\title{
Investigation of the Neuropathic Pain Caused by Syringomyelia Associated with Chiari I Malformation
}

\author{
Toshitaka Seki ${ }^{1}$, Shuji Hamauchi ${ }^{1}$, Masayoshi Yamazaki ${ }^{1}$, \\ Kazutoshi Hida ${ }^{2}$, Shunsuke Yano ${ }^{2}$, Kiyohiro Houkin ${ }^{1}$ \\ ${ }^{I}$ Department of Neurosurgery, Hokkaido University Graduate School of Medicine, Sapporo, Japan \\ ${ }^{2}$ Department of Neurosurgery, Sapporo Azabu Neurosurgical Hospital, Sapporo, Japan
}

\section{Study Design: Retrospective cohort study.}

Purpose: To investigate the correlation between the syrinx morphology and neuropathic pain caused by syringomyelia associated with Chiari I malformation.

Overview of Literature: Neuropathic pain caused by syringomyelia is refractory and markedly impairs the patient.

Methods: We examined 24 patients with neuropathic pain caused by syringomyelia associated with Chiari I malformation. We statistically analyzed the illness duration and age at surgery between patients with and without neuropathic pain. Additionally, we classified the morphology of the syringes into deviated (D), enlarged (E), central (C), and bulkhead (B) types using T2-weighted axial imaging. Moreover, we investigated the correlation between syrinx morphology and neuropathic pain. A Mann-Whitney U-test was performed to compare between the presence or absence of neuropathic pain and the presence or absence of type $D$ syringes.

Results: The median age at surgery was 27.5 years, and the median illness duration was 24 months. Among the 24 patients, 11 had preoperative neuropathic pain, one of which was free of neuropathic pain during the final follow-up period. Among patients with neuropathic pain, the syringes' preoperative morphology was type $D$ in nine patients and types $E$ and $C$ in one patient each. No patient exhibited type $B$ morphology. Among patients without neuropathic pain, the preoperative morphology of the syringes was type $D$ in three patients, type $E$ in seven patients, and types $C$ and $B$ in two patients each. For types $D$ and $E$, a correlation between neuropathic pain and syrinx morphology was observed. Moreover, type $\mathrm{D}$ was associated with significant neuropathic pain in both preoperative and postoperative states.

Conclusions: This study showed a correlation between the morphological features of the syringes and the occurrence of neuropathic pain in patients with syringomyelia associated with Chiari I malformation.

Keywords: Chiari malformation type 1; Magnetic resonance imaging; Neuralgia; Syringomyelia

\section{Introduction}

Syringomyelia associated with Chiari malformation type
I produces various symptoms, including motor deficits and sensory disturbances. Particularly, neuropathic pain is refractory and markedly impairs the patient's daily life.

Received Sep 18, 2018; Revised Nov 6, 2018; Accepted Nov 10, 2018

Corresponding author: Toshitaka Seki

Department of Neurosurgery, Hokkaido University Graduate School of Medicine, N-15, W-7, Kita-ku, Sapporo, 0608638, Hokkaido, Japan

Tel: +81-11-706-5987, Fax: +81-11-708-7737, E-mail: toseki1@hotmail.com 
We investigated the relationship between the preoperative morphological features of the syringes and the occurrence of neuropathic pain.

\section{Materials and Methods}

We explained the necessity of this research and obtained prior informed consent from all patients. Twenty-four patients underwent surgery for syringomyelia associated with Chiari I malformation at the Hokkaido University Hospital, between 2005 and 2016. The patients comprised six males and 18 females, whose age at the time of surgery ranged from 6 to 59 years (mean, 27.3 years; median, 27.5 years). Sixteen patients underwent foramen magnum decompression and $\mathrm{C} 1$ laminectomy along with incision of the dura mater's outer layer [1], seven patients underwent placement of a syringosubarachnoid shunt, and one patient underwent placement of a lumboperitoneal shunt. The patients were divided into two groups according to the presence or absence of preoperative neuropathic pain. The overall parameters examined were the age at the time of surgery, the disease duration, and the morphological features of the syringes based on preoperative magnetic resonance (MR) images. The syrinx morphology was classified into the following four types: central (C); enlarged (E); deviated (D); and bulkhead (B) based on the axial T1weighted MR imaging findings at the corresponding level of the dermatomal distribution of pain in patients experiencing pain and at the level corresponding to the maximal syrinx size in patients without neuropathic pain (Fig. 1).

A total of 24 patients were included in this study, 11 with and 13 without neuropathic pain. However, because one patient without neuropathic pain exhibited two types of syrinx at the maximal level, the analysis was made on 14 syringes without neuropathic pain.

Additionally, the correlation between syrinx morphology and the occurrence of neuropathic pain was investigated (Table 1). The Mann-Whitney $U$-test was performed to evaluate the preoperative syrinx morphology (i.e., type $\mathrm{D}$ or not) between patients with and without preoperative neuropathic pain. Moreover, the Mann-Whitney $U$-test was performed to evaluate the postoperative syrinx morphology (i.e., type D or not) between patients with and without postoperative neuropathic pain. A $p$-value of $<0.05$ was considered statistically significant. All analyses were performed using Ekuseru-Toukei 2015 (Social Survey Research Information Co. Ltd., Tokyo, Japan).
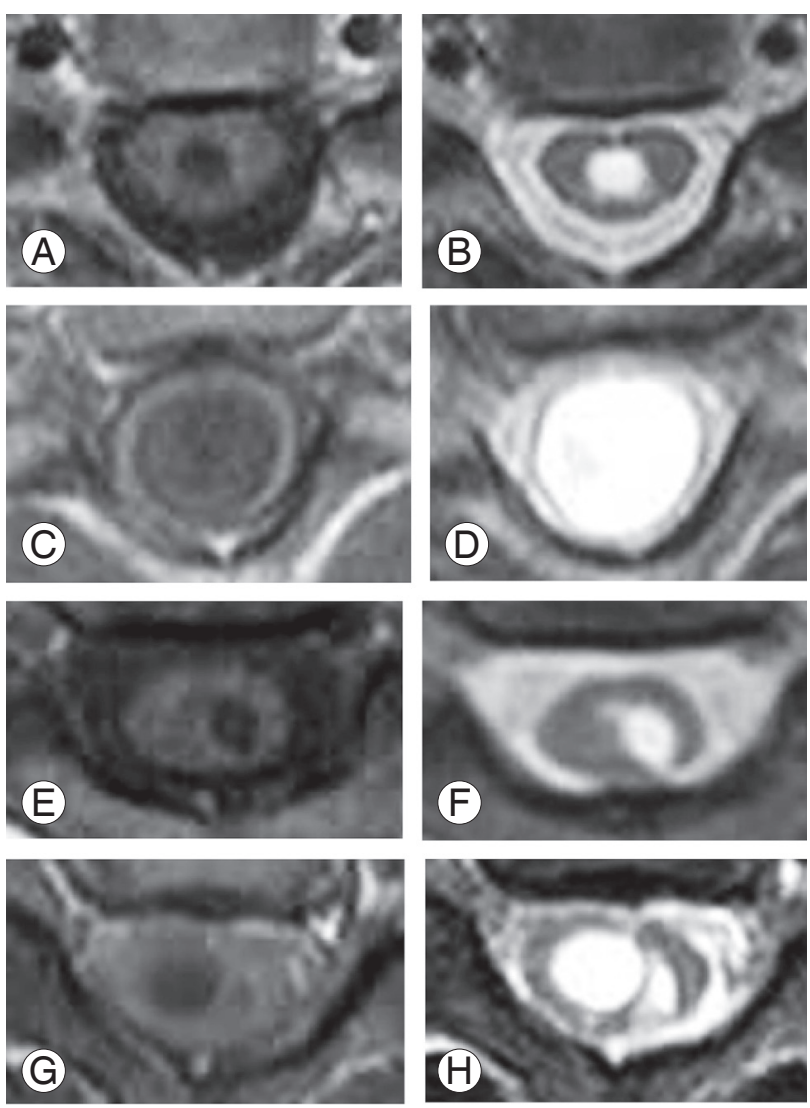

Fig. 1. Classification of the syrinx type. The syringes' morphological features were classified into four types (i.e., central type, enlarged type, deviated type, and bulkhead type) based on axial magnetic resonance images from the level corresponding to the dermatomal distribution of pain in patients with neuropathic pain and from the level of maximal syringe size in patients without neuropathic pain. (A, B) Central type: T1weighted image (A) and T2-weighted image (B). (C, D) Enlarged type: T1-weighted type (C) and T2-weighted type (D). (E, F) Deviated type: T1-weighted type (E) and T2-weighted type (F). (G, H) Bulkhead type: T1-weighted type (G) and T2-weighted type (H).

Table 1. Indication of correlation

\begin{tabular}{lc} 
Correlation & Correlation coefficient \\
\hline Slight correlation & $0.0 \pm 0.2$ \\
\hline Moderate correlation & $0.2 \pm 0.4$ \\
\hline Correlation & $0.4 \pm 0.7$ \\
\hline Strong correlation & $0.7 \pm 0.9$ \\
\hline Very strong correlation & $0.9 \pm 1.0$ \\
\hline
\end{tabular}

\section{Results}

The median age at the time of surgery was 28 years (range, 7-50 years) among patients with preoperative neuropathic pain and 19 years (range, 6-59 years) among patients 
without preoperative neuropathic pain. There was no significant difference in age between the two groups $(p=0.27)$. The median disease duration was 17 months (range, 2-120 months) among patients with preoperative neuropathic pain and 32.5 months (range, 4-204 months) among patients without preoperative neuropathic pain. There was no significant difference in the disease duration between the two groups $(p=0.17)$. Neuropathic pain location was the chest in six patients (46\%), the upper extremity in 10 patients (77\%), and the scapula's surroundings in one patient (33\%). Morphological feature's analysis of the syrinx in patients with and without preoperative neuropathic pain revealed type $\mathrm{C}$ in three patients (12\%), type $\mathrm{E}$ in eight patients (32\%), type $\mathrm{D}$ in 12 patients (48\%), and type B in two patients (8\%) (Table 2). One patient without neuropathic pain showed both $\mathrm{C}$ - and E-type syrinx preoperatively and at a completely different level. Among patients with preoperative neuropathic pain, only one experienced neuropathic pain relief during the final follow-up period. In this patient, the syrinx morphology changed from type $\mathrm{D}$ to type $\mathrm{C}$. Table 3 presents the postoperative changes in the syringes' morphological features among the patients with preoperative neuropathic pain. All patients without preoperative neuropathic pain remained pain free in the final follow-up period. Table 4 presents the postoperative changes in the syringes' morphological features among the patients without preoperative neuropathic pain.

Regarding the correlation between syrinx morphology and neuropathic pain during the preoperative period, a positive correlation was found between the occurrence of neuropathic pain and type D syrinx (correlation coefficient, $0.60 ; p=0.003$ ), whereas a negative correlation was found between the occurrence of neuropathic pain

Table 2. Correlation between morphological features of syrinx and preoperative neuropathic pain

\begin{tabular}{lccc} 
Morphological & \multicolumn{3}{c}{ Preoperative neuropathic pain } \\
\cline { 2 - 4 } features of syrinx & Present & Absent & Total \\
\hline Central type & 1 & 2 & 3 \\
\hline Enlarged type & 1 & 7 & 8 \\
\hline Deviated type & 9 & 3 & 12 \\
Bulkhead type & 0 & 2 & 2 \\
\hline Total & 11 & 14 & 25 \\
\hline
\end{tabular}

Values are presented as number of patients.

Table 3. Postoperative changes in morphological features of syrinx in patients with preoperative neuropathic pain

\begin{tabular}{|c|c|c|c|c|c|c|}
\hline \multirow{2}{*}{ Preoperative status } & \multicolumn{6}{|c|}{ Postoperative status } \\
\hline & Central type & Enlarged type & Deviated type & Bulkhead type & Disappearance & Total \\
\hline Central type & & 1 & & & & 1 \\
\hline Enlarged type & & & & & 1 & 1 \\
\hline Deviated type & 1 & & 6 & & 2 & 9 \\
\hline Bulkhead type & & & & & & \\
\hline Total & 1 & 1 & 6 & & 3 & 11 \\
\hline
\end{tabular}

Values are presented as number of patients.

Table 4. Postoperative changes in morphological features of syrinx inpatients without preoperative neuropathic pain

\begin{tabular}{|c|c|c|c|c|c|c|}
\hline \multirow{2}{*}{ Preoperative status } & \multicolumn{6}{|c|}{ Postoperative status } \\
\hline & Central type & Enlarged type & Deviated type & Bulkhead type & Disappearance & Total \\
\hline Central type & 1 & & & & 1 & 2 \\
\hline Enlarged type & 2 & 2 & & & 3 & 7 \\
\hline Deviated type & & 1 & & 2 & & 3 \\
\hline Bulkhead type & & & & 2 & & 2 \\
\hline Total & 3 & 3 & & 4 & 4 & 14 \\
\hline
\end{tabular}

Values are presented as number of patients. 
and type E syrinx (correlation coefficient, $-0.4 ; p=0.03$ ). Only a slight correlation was found between type B syrinx and the occurrence of neuropathic pain (correlation coefficient, $-0.26 ; p=0.2$ ), and no correlation was found between the occurrence of neuropathic pain and type $C$ syrinx (correlation coefficient, $-0.08 ; p=0.70$ ).

A comparison between patients with and without preoperative neuropathic pain revealed a significantly

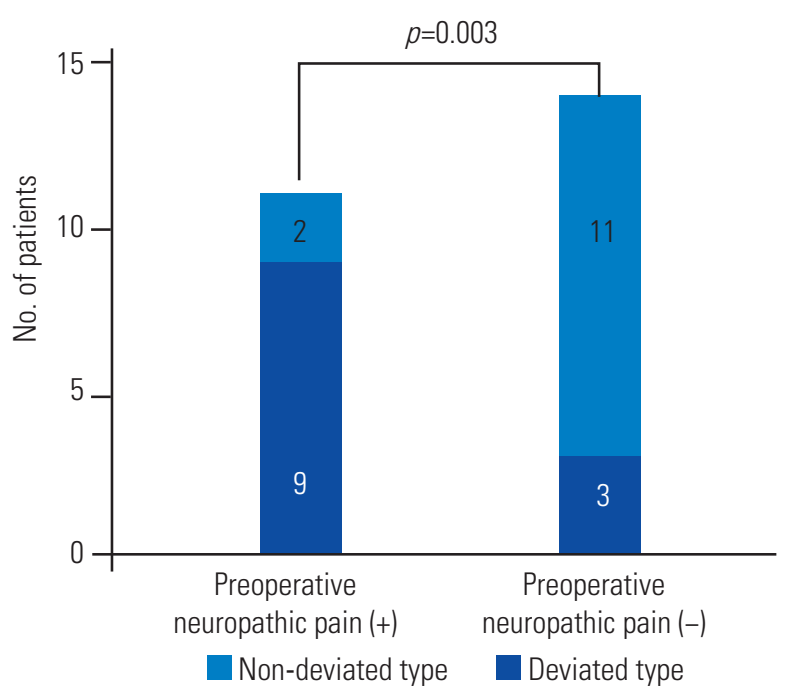

Fig. 2. The graph shows the comparison between patients with and without preoperative neuropathic pain. Patients with preoperative neuropathic pain exhibited a significantly higher occurrence of deviated type of syrinx $(p=0.003)$

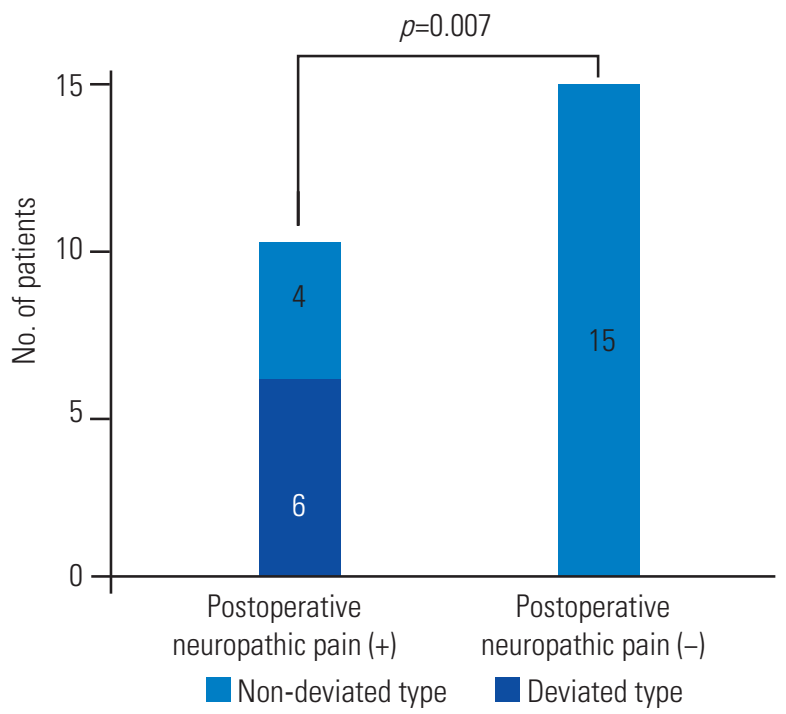

Fig. 3. The graph shows the comparison between patients with and without postoperative neuropathic pain. Patients with postoperative neuropathic pain exhibited a significantly higher occurrence of deviated type of syrinx. No deviated type of syrinx was observed in patients without neuropathic pain $(p=0.007)$. higher occurrence of type D syrinx among patients with preoperative neuropathic pain $(p=0.003)$ (Fig. 2). Similarly, a comparison between patients with and without postoperative neuropathic pain revealed a significantly higher occurrence of type D syrinx among patients with postoperative neuropathic pain. Conversely, type D syrinx was not found among patients without neuropathic pain ( $p=0.007)$ (Fig. 3).

\section{Discussion}

Syringomyelia is a chronic disorder of the spinal cord, characterized by the presence of spinal cord cavitation. It has multiple causes, such as trauma, intramedullary tumors, and degenerative diseases. It has several classifications and occurs most commonly in association with Chiari I malformation. In general, the surgical treatment of syringomyelia associated with Chiari I malformation is achieved through either foramen magnum decompression or syringosubarachnoid shunting $[1,2]$. Those surgical techniques aim to reduce the size of the syrinx by improving the circulation of the cerebrospinal fluid. However, neurological symptoms do not always improve, despite the syrinx's size reduction. Syringomyelia causes various symptoms such as numbness, muscle atrophy, autonomic disorders, and neuropathic pain. Among those, neuropathic pain within the body or in the upper or lower extremities exerts a particularly devastating effect on the patients' quality of life.

Few reports have addressed the relationship between the syrinx's morphological features and the occurrence of neuropathic pain [3-5]. Ono et al. [5] showed that in patients with type D syrinx, the upper extremity dermatome level, at which the pain was felt, corresponded with the spinal cord level, at which the syrinx deviated. Furthermore, they reported that both preoperative and postoperative pain was more intense when the syrinx deviated toward the spinal dorsal horn, as observed on MR imaging. Similarly, Nakamura et al. [4] found that in patients in whom preoperative MR imaging revealed a syrinx deviated toward the posterolateral aspect of the spinal cord at the level corresponding to the dermatomal distribution of the pain, the syrinx often remained at the same site postoperatively, even if it had reduced in size. The improvement in pain was therefore poor. In the present study, the most common syrinx type among patients with preoperative neuropathic pain was type D (82\%). Moreover, only 
$20 \%$ of the patients without preoperative neuropathic pain had a type D syrinx, and patients without postoperative neuropathic pain did not have type D syringes.

Rexed [6,7] focused on the structural difference of neurons and divided the gray columns of the spinal cord into 10 layers. According to this division, the first to sixth layers constitute the dorsal horn of the spinal cord. Both $\mathrm{A} \delta$ and $\mathrm{C}$ fibers, which are nociceptive fibers, bind synaptically only to the first layer and overexcite the second neuron in the spinothalamic pathway [8-10]. Furthermore, tracts also course into the spinothalamic tract via the third and fourth layers [10], and the fibers from the fourth and fifth layers project into the brain stem's reticular formation. These projection fibers are thought to play an essential role in the maintenance of homeostasis at the time of pain sensation [10]. Both in the preoperative and postoperative periods of the present study, a significant number of patients with neuropathic pain showed a type D syrinx, suggesting that the posterior horn of the spinal cord, especially the first layer, was irreversibly destroyed in most patients with a type $\mathrm{D}$ syrinx.

Additionally, three patients with preoperative neuropathic pain showed syrinx disappearance, although they continued to present neuropathic pain postoperatively. On the other hand, one patient with preoperative neuropathic pain before surgery experienced postsurgical pain relief, and her syrinx remained after surgery, changing from type $\mathrm{D}$ to type $\mathrm{C}$. This result also suggests that if disorder of the dorsal horn by syrinx was reversible, neuropathic pain is likely to disappear.

This study has some limitations. First, the number of patients with syringomyelia associated with Chiari I malformation was small, making it difficult to accurately assess the differences between neuropathic and non-neuropathic pain, in terms of the morphological features of the syrinx. Another limitation is the retrospective nature of the study, which prevented the formulation of conclusions on causality. Prospective studies are therefore required to confirm the conclusions of the present study.

\section{Conclusions}

The present study evaluated neuropathic pain and syrinx morphology. No significant differences were found between patients with and without neuropathic pain, regarding the age at the time of surgery and the duration of the disease. Moreover, in patients with type D syringes, a positive correlation was found between the syringes' morphology and the occurrence of preoperative neuropathic pain. Oppositely, a negative correlation was found in patients with type E syringes. Type D syringes were found to be significantly associated with neuropathic pain. However, the study included only 24 patients, which constitutes its major limitation.

\section{Conflict of Interest}

No potential conflict of interest relevant to this article was reported.

\section{Acknowledgments}

We thank Angela Morben, DVM, ELS, from Edanz Group (http://www.edanzediting.com/ac), for editing a draft of this manuscript.

\section{References}

1. Isu T, Sasaki H, Takamura H, Kobayashi N. Foramen magnum decompression with removal of the outer layer of the dura as treatment for syringomyelia occurring with Chiari I malformation. Neurosurgery 1993;33:845-9.

2. Hida K, Iwasaki Y, Koyanagi I, Sawamura Y, Abe H. Surgical indication and results of foramen magnum decompression versus syringosubarachnoid shunting for syringomyelia associated with Chiari I malformation. Neurosurgery 1995;37:673-8.

3. Milhorat TH, Kotzen RM, Mu HT, Capocelli AL Jr, Milhorat RH. Dysesthetic pain in patients with syringomyelia. Neurosurgery 1996;38:940-6.

4. Nakamura M, Chiba K, Nishizawa T, Maruiwa $H$, Matsumoto M, Toyama Y. Retrospective study of surgery-related outcomes in patients with syringomyelia associated with Chiari I malformation: clinical significance of changes in the size and localization of syrinx on pain relief. J Neurosurg 2004;100:241-4.

5. Ono A, Numasawa T, Wada K, et al. Surgical outcomes of foramen magnum decompression for syringomyelia associated with Chiari I malformation: relation between the location of the syrinx and body pain. J Orthop Sci 2010;15:299-304.

6. Rexed B. The cytoarchitectonic organization of the spinal cord in the cat. J Comp Neurol 1952;96:414-95. 
7. Rexed B. A cytoarchitectonic atlas of the spinal cord in the cat. J Comp Neurol 1954;100:297-379.

8. Andrew D, Craig AD. Quantitative responses of spinothalamic lamina I neurones to graded mechanical stimulation in the cat. J Physiol 2002;545:913-31.
9. Craig AD, Andrew D. Responses of spinothalamic lamina I neurons to repeated brief contact heat stimulation in the cat. J Neurophysiol 2002;87:1902-14.

10. Craig AD. Pain mechanisms: labeled lines versus convergence in central processing. Annu Rev Neurosci 2003;26:1-30. 